Nota de pesquisa/ Research note

\title{
OBSERVAÇÕES PRELIMINARES SOBRE A ATRATIVIDADE POR DIFERENTES CORES EM CALLIPHORIDAE (DIPTERA), NITERÓI, RJ, BRASIL
}

\section{Mariana Borges Fraga \& José Mario d‘Almeida}

\begin{abstract}
Preliminary remarks on attractiveness to different colours in Calliphoridae (Diptera), Niterói, RJ, Brazil - This research aimed to evaluate the influence of colours on the collecting of Calliphoridae flies. The methodology was based on colour traps (green, black, yellow, red, blue and white), baited with fish rotten (sardine). Green, black and red colours were the most attractive to the blowflies. It were collected 3549 flies, belonging to nine species. The most frequent were Chrysomya megacephala (Fabricius, 1794) $(74,86 \%)$ and Lucilia eximia (Wiedemann, 1819) $(14,06 \%)$.
\end{abstract}

Key words : Blowflies, Calliphoridae, colour traps

Laboratório de Bioecologia e Comportamento Animal, Departamento de Biologia Geral, Universidade Federal Fluminense, Outeiro de São João Batista s/n, CEP: 24020-150 Niterói, Rio de Janeiro, Brasil. E-mail: dalmeida@vm.uff.br

Recebido em: 06/12/2004.

Aceito em: 01/03/2005. 
Os dípteros muscóides, além da importância ecológica, por terem larvas que contribuem para a eliminação de carcaças, fezes e outros tipos de matéria orgânica em decomposição, também podem veicular mecanicamente uma série de patógenos, tais como: enterovírus, enterobactérias, cistos de protozoários e ovos de helmintos (Greenberg, 1976). As moscas são atraídas por uma série de substratos, que vão desde carcaças, fezes e frutas em decomposição (d'Almeida \& Lopes, 1983). Visando os levantamentos populacionais, variados tipos de armadilhas e de iscas têm sido testados (Lopes, 1973; Ferreira, 1978; Wall \& Smith, 1996; Oliveira et al., 2002). Contudo, são escassos os estudos que avaliam a atratividade exercida pelas cores (Lee, 1937; MacLeod \& Donnelly, 1956; Botelho et al., 1973; Lara et al., 1975; Jenkins \& Roques, 1993; Wall \& Smith, 1996). Portanto, o presente trabalho, teve por objetivo testar a atratividade que diferentes cores podem exercer sobre dípteros da família Calliphoridae.

As coletas foram efetuadas no Campus do Valonguinho da Universidade Federal Fluminense, que fica no bairro de São Domingos, Niterói, RJ. Encontra-se no Campus uma vegetação exuberante, com árvores frondosas, seculares, abrigando uma variada entomofauna. Para a captura das moscas utilizou-se armadilhas descritas por Ferreira (1978) com modificações, em que garrafas plásticas transparentes de refrigerante de 2 litros foram pintadas com tintas das seguintes cores: verde, preto, amarelo, vermelho, azul e branco. As armadilhas, em número de duas para cada cor, foram iscadas com peixe putrefato (sardinha), exposto previamente no meio ambiente durante 48 horas, em um mesmo recipiente, com o propósito de manter homogênea a decomposição do peixe utilizado. As armadilhas foram fixadas, lado a lado, em dois suportes semelhantes a traves, feitos com cano de PVC de $3 \mathrm{~cm}$ de diâmetro. Essas traves foram fincadas paralelamente no solo, com uma distância de 2 metros. Procurou-se também revezar a posição das armadilhas no suporte, evitando assim, que as moscas procurassem-nas, não pela atratividade exercida pelas cores, objetivo do presente trabalho, mas sim pelo condicionamento em relação ao local. O levantamento foi conduzido de primeiro de março a trinta de junho de 2004, sendo nesse período efetuadas 15 coletas. As armadilhas eram expostas às 9 horas e retiradas às 16 horas; as moscas coletadas eram mortas com éter, deixadas secar, sendo identificados apenas os califorídeos, que eram acondicionados em recipientes apropriados para serem processados posteriormente. Os exemplares tes- 
temunhos encontram-se depositados no Laboratório de Bioecologia e Comportamento Animal do Departamento de Biologia Geral da Universidade Federal Fluminense, Niterói, Rio de Janeiro.

Foram coletados 3549 califorídeos, pertencentes a nove espécies: Chrysomya megacephala (Fabricius, 1794); Lucilia eximia (Wiedemann, 1819); Chrysomya albiceps (Wiedemann, 1830); Hemilucilia segmentaria (Fabricius, 1805); Hemilucilia semidiaphana (Rondani, 1850); Chrysomya putoria (Wiedemann, 1830) Cochliomyia macellaria (Fabricius, 1805); Chloroprocta idioidea (Robineau-Devoidy, 1830) e Lucilia cuprina (Wiedemann, 1830) (Tab. I).

De um modo geral, as armadilhas verdes, seguidas pelas pretas e vermelhas, foram as que mais atraíram califorídeos $(24,48 \%, 18,08 \%$ e $17,30 \%$, respectivamente). Utilizou-se o peixe (sardinha) como isca, por se tratar de um substrato altamente atrativo para moscas, em especial nas áreas urbanas (d'Almeida \& Lopes, 1983). Nos meses de coleta, a temperatura média variou entre $22^{\circ} \mathrm{C}$ a $26^{\circ} \mathrm{C}$, e a precipitação entre $50 \mathrm{~mm}$ e $200 \mathrm{~mm}$ (fonte: Inmet). Dentre os muscóides capturados, $C$. megacephala foi o mais abundante $(74,86 \%)$. As cores que mais atraíram este califorídeo foram verde $(24,50 \%)$ e preto $(18,89 \%)$. As armadilhas brancas foram as menos atrativas (11,06\%). Quanto aos outros califorídeos deste gênero, C. albiceps $(5,29 \%)$, também foi mais atraída pelas cores verde $(25,53 \%)$ e preta $(24,46 \%)$.

Lucilia eximia foi a segunda espécie em abundância (14,06\%), tendo sido atraída com maior freqüência pelo vermelho e verde $(23,84 \%$ e $23,24 \%$, respectivamente). L. cuprina, abundante em outros levantamentos (d'Almeida \& Lopes, 1983), foi pouco freqüente (Tab. I). Segundo Wall \& Smith (1996), em condições naturais, as cores preferidas por Lucilia sericata (Meigen, 1826), espécie próxima à L. cuprina, foram: vermelho, branco, preto e amarelo, enquanto que, no laboratório, foi a amarela. No entanto, Hall (1995) observou que o preto foi a cor mais atrativa para $L$. sericata, esses autores chamaram a atenção para a interferência de diferentes iscas sobre a atratividade das cores.

Em um experimento de aprendizagem com dípteros muscóides, Fukushi (1989) observou que o azul e o amarelo foram discriminados com mais facilidade pela $L$. cuprina e, segundo esse mesmo autor, as cores vermelho e preto são as mais difíceis de serem discriminadas.

Os califorídeos do gênero Hemilucilia, representados no presente trabalho pela $H$. segmentaria $(3,35 \%)$ e $H$. semidiaphana $(0,87 \%)$ são pouco 


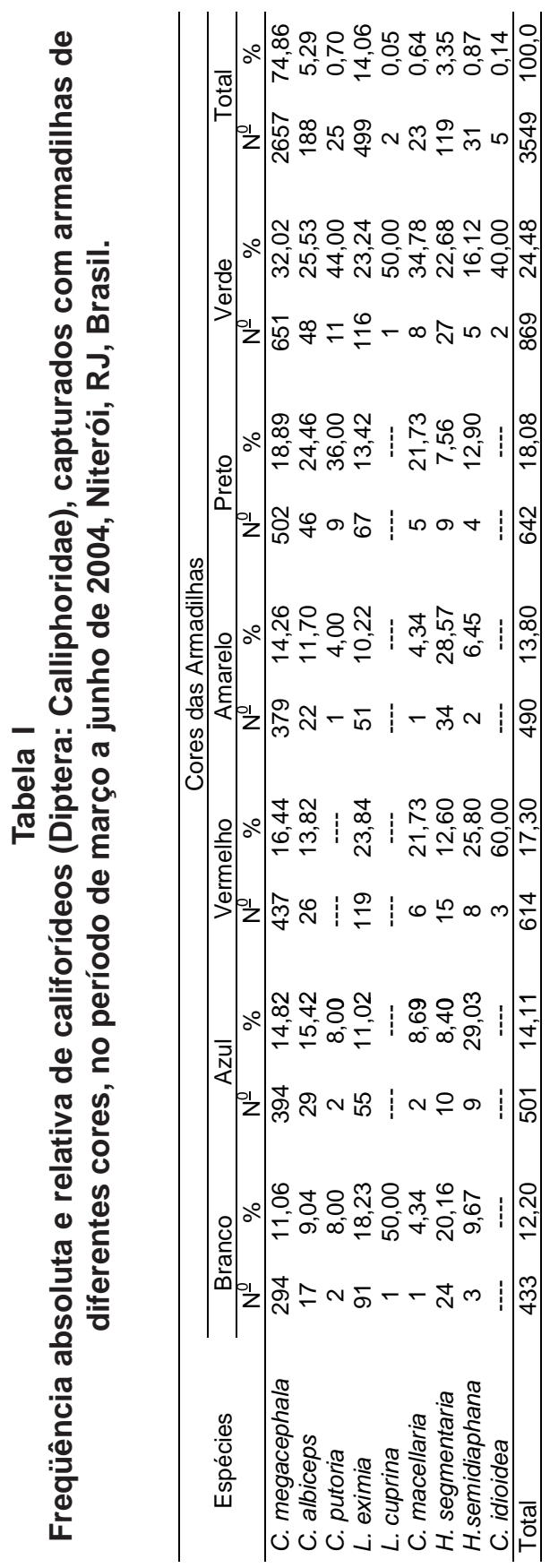


estudados. Esses muscóides são mais freqüentes nos ambientes silvestres e rurais, do que nos urbanos (d'Almeida \& Lopes, 1983). Hemilucilia segmentaria foi mais atraída pelo amarelo e verde, enquanto que, em $\mathrm{H}$. semidiaphana prevaleceram as cores azul e vermelho. Quanto às espécies restantes, devido ao pequeno número de exemplares coletados, não foram feitas maiores considerações, apenas pode-se chamar a atenção para a preferência dessas espécies pelas armadilhas vermelhas (Tab. I).

Como já foi ressaltado por Wall \& Smith (1996), diferentes iscas podem interferir na atratividade exercida pelas cores. Um outro fator a ser considerado é a incidência solar, tanto que, procurou-se distribuir as armadilhas em locais, onde pelo menos, durante uma parte do dia incidisse o sol. Lara et al. (1975) constataram que os muscídeos Musca domestica L., 1758 e Stomoxys calcitrans L., 1758 são mais atraídas pelo branco e amarelo, em pleno sol e pelo branco e azul, à sombra.

Ferreira (1978) e Linhares (1981) destacam que as armadilhas pintadas de preto são as que mais atraem dípteros muscóides. Entretanto, observou-se no presente trabalho que as verdes foram as mais atrativas. Contudo, é conveniente ressaltar que essas diferenças podem estar associadas a variados fatores, tais como: tipos de iscas, locais de coleta, claridade e etc. Na próxima etapa da pesquisa pretende-se avaliar alguns fatores que podem interferir no comportamento dos muscóides frente a diferentes cores.

\section{Referências Bibliográficas}

Botelho,P.S.N; Silveira-Neto, S; Salles, L.A B; Barbin, D. \& Borges, C.G., 1973. Testes de atração de Musca domestica L., com luzes de diferentes comprimentos de onda. Solo 65:42-45.

d'Almeida, J.M. \& Lopes, H.S., 1983. Sinantropia de Dípteros Muscóides (Calliphoridae) no Estado do Rio de Janeiro. Arq. Univ. Fed. Rur. RJ 6:39-48.

Ferreira, M.J.M., 1978. Sinantropia de dípteros muscóides de Curitiba, Paraná. I. Calliphoridae. Rev. Bras. Biol. 38:445-454. 
Fukushi, T. 1989. Learning and discrimination of coloured papers in the walking blowfly, Lucilia cuprina. J. Comp. Physyol. A. 166:57-64.

Greenberg, B., 1976. Flies and Diseases. Volume I. Princeton University Press. Princeton, N. J., 856 p.

Hall, M.J.R., 1995. Trapping the flies that cause myiasis; their responses to host stimuli. Ann. Trop. Med. Parasit. 89:333-357.

Jenkins, M.J. \& Roques, A., 1993. Attractiveness of collor traps to Strobilomyia (Diptera: Anthomyidae). Env. Entomol. 22:297-304.

Lara, F.M; Marchiori, D.L. \& Busoli, A.C.,1975. Atratividade de cores à Musca domestica L. e Stomoxys calcitrans (L.) (Díptera: Muscidae), a pleno sol e à sombra. Cientifica 3:73-80.

Lee, D.F., 1937. A note on the colour responses of Lucilia cuprina. J. Council Scien. Indust. Research 10:275-276.

Linhares, A.X., 1981. Sinanthropy of Calliphoridae and Sarcophagidae (Diptera) in the city of Campinas, São Paulo, Brazil. Rev. Bras. Entomol. 25:189-215.

Lopes, H.S., 1973. Collecting and rearing Sarcophagidae flies (Diptera) in Brazil, during forty years. An. Acad. Bras. Cien. 45:279-291.

Macleod, J. \& Donnelly, J., 1956. Methods for the study of blowfly populations. I. Bait trapping; significance limits for comparative sampling. Ann. Appl. Biol. 44:80-104.

Oliveira, V.C; d'Almeida, J.M; Paes, M.J. \& Sanavria, A., 2002. Population dynamics of Calyptrate Diptera (Muscidae and Sarcophagidae) at the RIO-ZOO Foundation, Rio de Janeiro, RJ, Brazil. Rev. Bras. Biol. 62:191-196.

Wall, R. \& Smith, K.E., 1996. Colour discrimination by the sheep blowfly Lucilia sericata. Med. Vet. Entomol. 10:235-240. 